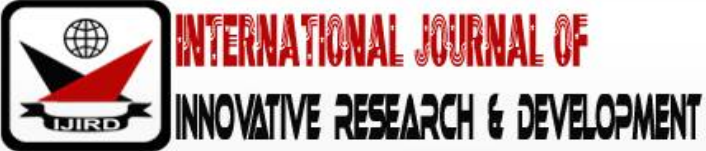

ISSN 2278 - 0211 (Online)

\section{Practical Analysis on the Effect of Shiruma Approach in Obtaining Optimal Preference Ordering in Multi-Person Fuzzy Decision Making}

\author{
Auwalu Sa'idu \\ Lecturer, Department of Mathematics, Yusuf Maitama Sule University, Kano, Nigeria
}

\begin{abstract}
:
This paper presents an analysis on the effect of Shiroma approach in data collection in multi-person decision making in fuzzy environment using a real-life situation.

Many at times decision(s) is/are made by more than one person and over many alternatives, as such this research work consider a group of 10 graduates on life carrier choice preference ordering over 5 alternatives, which are; Politics, Civil Service, Marriage, Farming and Business. These alternatives are considered because of the fact that they are the immediate possibilities for most of graduates and also, finding the best opinion(s) of our youths, with regard to them, will help in determining the direction in thoughts and attitudes of our graduates, as such a best technique needs to be employed.

In this paper, at first, based on Shiruma approach, the preference ordering of each individual is obtained and then, later, by Blin approach, where the opinions/goals of the individuals involved in the group are equally considered, the optimal preference ordering of the decision for the group is obtained. Later, the preference orderings of the individuals are collected directly and the group decision is calculated in similar way.

The two different results obtained were analysed and, provided the required conditions are considered, Shiruma approach is proved to be the best way in data collection in making decision in fuzzy environment.
\end{abstract}

Keywords: Fuzzy set, fuzzy decision, preference ordering, membership function, and optimal decision

\section{Introduction}

Making decisions is undoubtedly one of the most fundamental activities of human beings. We all are faced in our daily life with varieties of alternative actions available to us and, at least in some instances, we have to decide which of the available actions to take. The beginnings of decision making, as a subject of study, can be traced, presumably, to the late 18th century, when various studies were made in France regarding methods of election and social choice [14].

The subject of decision-making is, as the name suggests, the study of how decisions are actually made and how they can be made better or more successfully. Since these initial studies, decision making has evolved into a respectable and rich field of study, as such many theories and methods were developed.

Fuzzy decision theories attempt to deal with the vagueness and non-specificity inherent in human formulation of preferences, constraints, and goals [4]. Analysing the effect of any developed method that is dealing with the fuzziness in making decisions is deemed necessary in order to have best technique that can lead to optimal solution.

\section{Literature Review}

The attempts to justify whether all valid propositions can only be assigned the logical values true or false by the introduction of fuzzy sets by Zadeh [13] brought about fuzzy concepts. In 1965, Lotfi Zadeh introduced his seminal idea in a continuous-valued logic that he called fuzzy set theory.

As its name implies, the theory of Fuzzy Set is basically, a theory in which everything is a matter of degree. Professor Zimmermann's treatise [20,21,22] provides an affirmative answer to the question that whether the fuzzy set theory can deal better with uncertainty phenomenon or not. His comprehensive exposition of both the theory and its applications explains in clear terms the basic concepts underline the theory and how they relate to their classical counterparts.

Professor Zimmermann's treatise relates to the distinction between the concepts of Probability and possibility, with latter concept having a close connection with that of membership in a Fuzzy Set. The concept of possibility plays a particularly important role in the representation of meaning, in the management in expert system, and in applications of the theory of Fuzzy Sets to decision analysis [21].

As an extension to fuzzy sets, intuitionistic fuzzy set (IFS) was proposed by Atanassov [12] to handle the issues of membership, non-memberships and hesitation degrees of decision problems. The IFS was intended to be an extension of 
single membership of fuzzy set theory. However, the two memberships of IFS have some limitations particularly on the arithmetic addition of two memberships and also hesitation degree. To improve hesitation degree, author such as Zeng et al. $[18,19]$ proposed interval valued hesitant fuzzy sets and its arithmetic operations. For the two memberships of IFS, the focal point is on its arithmetic addition. It is known that the sum of two memberships of IFS is limited to one. In response to this limitation, Yager [17] introduced Pythagorean fuzzy set (PFS) where the limitation in IFS has been modified. The sum of two memberships in IFS is now substituted with squares of each membership where the sum of these two squares is less or equal to one. In other words, the PFS is characterized by a membership degree and non-membership degree where the square sum of its membership degree and non-membership degree is less than or equal to one. The PFS is one of the most successful sets, in terms of representing comprehensively uncertain and vague information [5]. The PFS also was successfully integrated with the concepts of confidence level [8], and decision making with probabilities [10]. The new linguistic and exponential operational laws with PFS were also proposed $[9,11]$. About similar with other sets, the PFS was also extended to interval-valued PFS and hesitant PFS. These two sets were successfully used in developing new aggregation operators such as Maclaurin Symmetric Mean Operator [7, 16], averaging and geometric aggregation operators [6].

In this paper, the analysis is based on the primary approach whereby only the degree of truth is taken into consideration and the process consider the normal aggregation operator by comparing one alternative over the other in order to obtained the preference ordering which resulted in determining the optimal preference ordering for the group of the decision makers.

\section{Definitions of Some Basic Terminologies}

Below are descriptions of some important terms that are used in this paper;

\subsection{Decision Theory}

Decision theory deals with methods for determining the optimal course of action when a number of alternatives are available and their consequences cannot be forecast with certainty. According to John Dewey [3], decision making is a problem-solving process that consists of five consecutive stages: a felt difficulty, the definition of the character of that difficulty, suggestion of possible solutions, evaluation of the suggestion, and further observation and experiment leading to acceptance or rejection of the suggestion.

\subsection{Classical Set Theory}

Mathematicians defined set as a collection of objects having one or more common characteristics. The objects that belong to a set are called members/elements of the set. Classical set theory is built on the fundamental concept of 'set' of which an individual is either a member or not a member. A sharp, crisp, and unambiguous distinction exists between a member and a non-member for any well-defined 'set' of entities in this theory.

\subsection{Fuzzy Set Theory}

A fuzzy set theory is an extension of the classical set theory where elements have varying degrees of membership. A fuzzy set is any set that allows its members to have different degree of membership function in the interval $[0,1]$.

A fuzzy set is described by a membership function $\mu_{A}(x)$ of $A$. This membership function associates each $x \in X$ a number $\mu_{A}(x)$ in the closed interval $[0,1]$. Below is a graphical descriptions of membership functions in classical case as well as in fuzzy case;

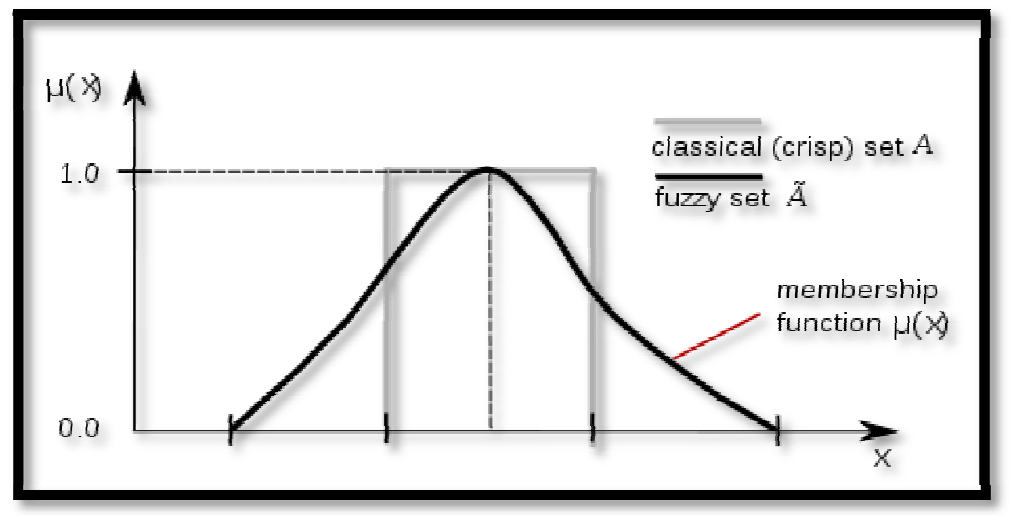

Figure 1: Graphical Representations of Membership Function in Crisp and Fuzzy Sets

\subsubsection{Definition of Fuzzy Set}

Let $X$ be a universal space (objects) with a generic element of $X$ denoted by $x$ (i.e. $X=\{x\}$ ) and $A$ be a fuzzy set in $X$, then a fuzzy set (class) $A$ in $X$ is a function defined in $X$ characterized by a membership function $\mu_{A}(x)$ which assigns to each point in $X$ a real number in the interval $[0,1]$, that is;

$A: X \rightarrow[0,1]$

A fuzzy set $A$ is defined by the membership function

$\mu_{A}(x): X \rightarrow[0,1]$ 
in addition, the value $\mu_{A}(x)$ represents the grade of membership of $x \in A$ and it is called membership value of $x \in X$.

NOTE: Each fuzzy set is defined by a unique membership function.

\subsubsection{Representation of a Fuzzy Set}

A fuzzy set $A$ consisting of elements $x_{i} \in X$, for $i=1,2,3 \ldots, n$ can be expressed as;

1. $\quad A=\left\{\left(x_{i}, \mu_{A}\left(x_{i}\right)\right) / x_{i} \in X, \mu_{A}\left(x_{i}\right): X \rightarrow[0,1]\right.$

2. $A=\left\{\frac{\mu_{A}\left(x_{1}\right)}{x_{1}}+\frac{\mu_{A}\left(x_{2}\right)}{x_{2}}+\ldots \ldots . .+\frac{\mu_{A}\left(x_{i}\right)}{x_{n}}\right\}=\sum_{i=1}^{n} \frac{\mu \mathrm{A}\left(x_{i}\right)}{x_{i}}$ and

3. by describing the nature/character of the elements of the set as in crisp case.

\subsection{Fuzzy Relation}

A fuzzy relation is a fuzzy set defined on the Cartesian product of crisp sets $A_{1}, A_{2}, A_{3}, \ldots, A_{n}$ where the $n$-tuples $\left(x_{1}, x_{2}, x_{3}, \ldots, x_{n}\right)$ may have varying degrees of membership within the relation.

The strength of the relation between the elements of the tuple is expressed by the membership function $\mu_{R}: A_{1} \times A_{2} \times A_{3} \times \ldots \times A_{n} \rightarrow[0,1]$

$\therefore R=\left\{\left(\left(x_{1} \times x_{2} \times x_{3} \times \ldots \times x_{n}\right), \mu_{R}\right) / \mu_{R}\left(x_{1}, x_{2}, x_{3}, \ldots, x_{n}\right) \geq 0, x_{1} \in A_{1}, x_{2} \in A_{2}, \ldots, x_{n} \in A_{n}\right\}$

$\boldsymbol{\alpha}$-Cut of Fuzzy Relation: Assume $R \subseteq A \times B$, and $\alpha_{\mathrm{R}}$ is a $\alpha$-cut relation then

$\alpha_{\mathrm{R}}=\left\{(x, y) / \mu_{R}(x, y), \mu_{R}(x, y) \geq \alpha, x \in A, y \in \mathrm{B}\right\}, \alpha \in[0,1]$

Therefore $\alpha$-cut of a fuzzy relation is always a crisp relation.

\subsubsection{Characteristics of Relation}

For a relation $R(X, Y)$ we have some characteristics as follows;

- Reflexive: $R(X, X)$ is reflexive if $x \in A, \Rightarrow(x, x) \in R, \forall x \in A$.

- Antisymmetric: $R(X, Y)$ is antisymmetric if $\forall x, y \in A,(x, y) \in R$ and $(y, x) \notin R$

- Transitive: $R(X, Y)$ is said to be transitive if $\forall x, y, z \in A,(x, y) \in R,(y, z) \in R$ then $(x, z) \in R$.

Order Relation: A Relation $\mathrm{R} \subset \mathrm{X} \times \mathrm{X}$ is said to be order relation if it is Reflexive, Antisymmetric and Transitive.

\subsection{Fuzzy Multiperson Decision Making}

When decisions made by more than one person are modeled, two cases can be considered: first, the goals of individual decision makers may differ such that each places a different ordering on the alternative, second, the individual decision makers may have access to different information upon which to base their decision. It can easily be seen that for the decision maker to passed a better decision, in this case, he/she must consider the uncertainty involved and the best way to, appropriately, capture such uncertainties is by using fuzzy decision-making approach.

\section{Methodology}

The approaches considered in this research are explained below and, later, the analysis is given based on the obtained data. The first letter(s) of the alternatives, for simplicity, are considered, that is: Politics (P), Civil Service (CS), Business (B), Marriage (M) and Farming (F).

\subsection{Shiroma Approach}

Shiroma approach is designed to construct an ordering of all given alternatives on the basis of their pairwise comparisons instead of direct preferring. This approach gives the strength of the preference of one option over the other. In this approach, $f\left(x_{i}, x_{j}\right)$ denotes the desirability grade given by the individual to $x_{i}$ with respect to $x_{j}$. These evaluations, which are expressed by positive numbers in a given range, are made by the individual for all pairs of alternatives in the given set $X$. They are then converted to relative preference grades, $F\left(x_{i}, x_{j}\right)$, by the formula

$$
F\left(x_{i}, x_{j}\right)=\frac{f\left(x_{i}, x_{j}\right)}{\max \left[f\left(x_{i}, x_{j}\right), f\left(x_{j}, x_{i}\right)\right]}=\min \left[1, \frac{f\left(x_{i}, x_{j}\right)}{f\left(x_{j}, x_{i}\right)}\right]
$$

For each pair $\left(x_{i}, x_{j}\right) \in X^{2}$. Clearly, $F\left(x_{i}, x_{j}\right) \in[0,1]$ for all pairs $\left(x_{i}, x_{j}\right) \in X^{2}$. When $f\left(x_{i}, x_{j}\right)=1, x_{i}$ is considered at least as desirable as $x_{j}$. Functions $F$, which stand as the membership function of a fuzzy relation on $X$, has for each pair the property that; $\max \left[f\left(x_{i}, x_{j}\right), f\left(x_{j}, x_{i}\right)\right]=1$.

Then, for each $x_{i} \in X$, the overall relative preference grades $P\left(x_{i}\right)$, of $x_{i}$ with respect to all other alternatives in $X$ is calculated by the formula:

$P\left(x_{i}\right)=\min _{x_{i} \in X} F\left(x_{i}, x_{j}\right)$

The preference ordering of each of the alternatives in $X$ is then induced by the numerical ordering of these grades $P\left(x_{i}\right)$ [15]. This gives the preference ordering for each of the members in the group.

\subsection{Blin Approach}

A fuzzy model group decision was proposed by Blin [1] and Blin and Whinstone [2]. Here, each member of a group of $n$ individual decision makers is assumed to have a reflexive, antisymmetric and transitive preferences ordering $P_{k}, k \in N$ which totally or partially ordered a set $X$ of alternatives. A 'social choice' function is then found which, given the individual 
preferences orderings, produces the most acceptable overall group preference orderings. In order to deal with the multiplicity of opinion evidenced in the group, the social preference $S$ is defined as a fuzzy binary relation with membership grade function

$$
S: X \times X \rightarrow[0,1]
$$

which assigns the membership grade $S\left(x_{i}, x_{j}\right)$, indicating the degree of group preference of alternative $x_{i}$ over $x_{j}$. The appropriate means of aggregating the individual preferences of this group requires computing the relative popularity of alternative $x_{i}$ over $x_{j}$ by dividing the number of persons preferring $x_{i}$ to $x_{j}$, denoted by $N\left(x_{i}, x_{j}\right)$, by the total number of decision makers, $n$. Thus,

$S\left(x_{i}, x_{j}\right)=\frac{N\left(x_{i}, x_{j}\right)}{n}$

Once the fuzzy relationship $S$ has been defined, the final nonfuzzy group preference can be determined by converting $S$ into its resolution form

$$
S=\bigcup_{\alpha \in[0,1]} \alpha^{\alpha} S
$$

which is the union of the crisp relations ${ }^{\alpha} S$ comprising the $\alpha$ - cuts of the fuzzy relation $S$, each scaled by $\alpha$. Each value $\alpha$ essentially represents the level of agreements between the individual concerning the particular crisp ordering ${ }^{\alpha} S$, to maximize the final agreement level, the intersection of the classes of crisp total ordering that are compatible with the pairs in the $\alpha$-cuts ${ }^{\alpha} S$ is considered for increasingly smaller values of $\alpha$ until a single crisp total ordering is achieved. In this processed, any pairs $\left(x_{i}, x_{j}\right)$ that lead to an intransitivity are removed. The largest value $\alpha$, for which the unique compatible ordering on $X \times X$ is found, represents the maximized agreement level of the group, and the crisp ordering itself represents the group decision [1,2].

\section{Calculating the Individual Preference Ordering}

In order to apply the above descriptions to construct an ordering of all the alternatives in consideration, on the basis of their pairwise comparisons, a questionnaire was designed and the data was obtained and processed. Let refer each of the $n$ individuals involved in making this decision by $P K$ while the corresponding set obtained by $P_{k}$, $k \in N$. As the number of the graduate is 10 , then $n=10$. The table below shows the descriptions used to obtained the data;

\begin{tabular}{|c|c|c|c|}
\hline $\boldsymbol{f}\left(\boldsymbol{x}_{\boldsymbol{i}}, \boldsymbol{x}_{\boldsymbol{j}}\right)$ & Desirability of $\boldsymbol{x}_{\boldsymbol{i}}$ over $\boldsymbol{x}_{\boldsymbol{j}}$ & $\boldsymbol{f}\left(\boldsymbol{x}_{\boldsymbol{i}}, \boldsymbol{x}_{\boldsymbol{j}}\right)$ & Desirability of $\boldsymbol{x}_{\boldsymbol{i}}$ over $\boldsymbol{x}_{\boldsymbol{j}}$ \\
\hline 1 & Little Desirable & 6 & Between 5 and 7 \\
\hline 2 & Between 1 and 3 & 7 & Desirable Very strongly \\
\hline 3 & Desirable Moderately & 8 & Between 7 and 9 \\
\hline 4 & Between 3 and 5 & 9 & Extremely Desirable \\
\hline 5 & Desirable Strongly & \multicolumn{3}{c}{} \\
\hline
\end{tabular}

Table 1: Suggested Numbers for Desirability Grading

Each of the individuals provided his desirability preference by pairwise comparisons of one alternative over the other on a table using the numbers suggested in the table 1 for specifying the desirability grades, the evaluating prepared by $\mathrm{P} 1$ (person number 1 ) is given in table 2 below. The corresponding relative preference grades (calculate by (1)) and the overall relative preference grades calculated by (calculated by (2)) are given in the table 3 . The overall relative preference induced the preference ordering of the alternatives.

\begin{tabular}{|c|c|c|c|c|c|}
\hline $\boldsymbol{f}\left(\boldsymbol{x}_{\boldsymbol{i}}, \boldsymbol{x}_{\boldsymbol{j}}\right)$ & $\mathbf{P}$ & $\mathbf{C S}$ & $\mathbf{B}$ & $\mathbf{M}$ & $\mathbf{F}$ \\
\hline $\mathrm{P}$ & 0 & 1 & 3 & 4 & 8 \\
\hline $\mathrm{CS}$ & 8 & 0 & 6 & 6 & 6 \\
\hline $\mathrm{B}$ & 1 & 4 & 0 & 8 & 5 \\
\hline $\mathrm{M}$ & 2 & 1 & 5 & 0 & 7 \\
\hline $\mathrm{F}$ & 5 & 9 & 6 & 6 & 0 \\
\hline
\end{tabular}

Table 2: The Desirability Grades Prepared by $P 1$

Calculating the Relative Preference Grades for P1;

For $i=1$ and $j=1,2,3,4$,or 5

$F\left(x_{1}, x_{1}\right)=\frac{1}{\max [1,1]}=1, F\left(x_{1}, x_{2}\right)=\frac{1}{\max [1,8]}=0.125$

$F\left(x_{1}, x_{3}\right)=\frac{3}{\max [3,1]}=1, F\left(x_{1}, x_{4}\right)=\frac{4}{\max [4,2]}=1, F\left(x_{1}, x_{5}\right)=\frac{8}{\max [8,5]}=1$

For $i=2$ and $j=1,2,3,4$ or 5

$F\left(x_{2}, x_{1}\right)=\frac{8}{\max [8,1]}=1, F\left(x_{2}, x_{2}\right)=1, F\left(x_{2}, x_{3}\right)=\frac{6}{\max [6,4]}=1$

$F\left(x_{2}, x_{4}\right)=\frac{6}{\max [6,1]}=1, F\left(x_{2}, x_{5}\right)=\frac{6}{\max [6,9]}=0.667$ 
For $i=3$ and $j=1,2,3,4$, or 5

$F\left(x_{3}, x_{1}\right)=\frac{1}{\max [1,3]}=0.333, F\left(x_{3}, x_{2}\right)=\frac{4}{\max [4,6]}=0.667, F\left(x_{3}, x_{3}\right)=1$

$F\left(x_{3}, x_{4}\right)=\frac{8}{\max [8,5]}=1, F\left(x_{3}, x_{5}\right)=\frac{5}{\max [5,6]}=0.833$

For $i=4$ and $j=1,2,3,4$,or 5

$F\left(x_{4}, x_{1}\right)=\frac{2}{\max [2,4]}=0.5, F\left(x_{4}, x_{2}\right)=\frac{1}{\max [1,6]}=0.167$

$F\left(x_{4}, x_{3}\right)=\frac{5}{\max [5,8]}=0.625, F\left(x_{4}, x_{4}\right)=1, F\left(x_{4}, x_{5}\right)=\frac{7}{\max [7,6]}=1$

For $i=5$ and $j=1,2,3,4$, or 5

$F\left(x_{5}, x_{1}\right)=\frac{5}{\max [5,8]}=0.625, F\left(x_{5}, x_{2}\right)=\frac{9}{\max [9,6]}=1$

$F\left(x_{5}, x_{3}\right)=\frac{6}{\max [6,5]}=1, F\left(x_{5}, x_{4}\right)=\frac{6}{\max [6,7]}=0.857, F\left(x_{5}, x_{5}\right)=1$

The relative preference grades are obtained as in the table below;

\begin{tabular}{|c|c|c|c|c|c|c|}
\hline $\boldsymbol{F}\left(\boldsymbol{x}_{\boldsymbol{i}}, \boldsymbol{x}_{\boldsymbol{j}}\right)$ & $\mathbf{P}$ & $\mathbf{C S}$ & $\mathbf{B}$ & $\mathbf{M}$ & $\mathbf{F}$ & $\boldsymbol{P}\left(\boldsymbol{x}_{\boldsymbol{i}}\right)$ \\
\hline $\mathrm{P}$ & 1.000 & 0.125 & 1.000 & 1.000 & 1.000 & 0.125 \\
\hline $\mathrm{CS}$ & 1.000 & 1.000 & 1.000 & 1.000 & 0.667 & 0.667 \\
\hline $\mathrm{B}$ & 0.333 & 0.667 & 1.000 & 1.000 & 1.000 & 0.333 \\
\hline $\mathrm{M}$ & 0.500 & 0.167 & 0.625 & 1.000 & 0.833 & 0.167 \\
\hline $\mathrm{F}$ & 0.625 & 1.000 & 1.000 & 0.857 & 1.000 & 0.625 \\
\hline
\end{tabular}

Table 3: Relatives Preference Grades and Overall Relative Preference Grades of P1

Now, from the above table the fuzzy set below is obtained;

$\mathrm{P}_{1}=\{\mathrm{CS} / 0.667, \mathrm{~F} / 0.625, \mathrm{~B} / 0.333, \mathrm{M} / 0.167, \mathrm{P} / 0.125\}$

The ordering expressions by the 9 other members of the group are determined in similar way and the following results/fuzzy sets are obtained

\begin{tabular}{|c|c|c|c|c|c|}
\hline $\boldsymbol{f}\left(\boldsymbol{x}_{\boldsymbol{i}}, \boldsymbol{x}_{\boldsymbol{j}}\right)$ & $\mathbf{P}$ & $\mathbf{C S}$ & $\mathbf{B}$ & $\mathbf{M}$ & $\mathbf{F}$ \\
\hline $\mathrm{P}$ & 0 & 2 & 3 & 5 & 6 \\
\hline $\mathrm{CS}$ & 9 & 0 & 6 & 7 & 6 \\
\hline $\mathrm{B}$ & 7 & 5 & 0 & 5 & 9 \\
\hline $\mathrm{M}$ & 8 & 2 & 4 & 0 & 5 \\
\hline $\mathrm{F}$ & 5 & 7 & 6 & 6 & 0 \\
\hline
\end{tabular}

Table 4: The Desirability Grades Prepared by P2

\begin{tabular}{|c|c|c|c|c|c|c|}
\hline $\boldsymbol{F}\left(\boldsymbol{x}_{\boldsymbol{i}}, \boldsymbol{x}_{\boldsymbol{j}}\right)$ & $\mathbf{P}$ & $\mathbf{C S}$ & $\mathbf{B}$ & $\mathbf{M}$ & $\mathbf{F}$ & $\boldsymbol{P}\left(\boldsymbol{x}_{\boldsymbol{i}}\right)$ \\
\hline $\mathrm{P}$ & 1.000 & 0.222 & 0.429 & 0.625 & 1.000 & 0.222 \\
\hline $\mathrm{CS}$ & 1.000 & 1.000 & 1.000 & 1.000 & 0.857 & 0.857 \\
\hline $\mathrm{B}$ & 1.000 & 0.833 & 1.000 & 1.000 & 1.000 & 0.833 \\
\hline $\mathrm{M}$ & 1.000 & 0.286 & 0.800 & 1.000 & 0.833 & 0.286 \\
\hline $\mathrm{F}$ & 0.833 & 1.000 & 0.667 & 1.000 & 1.000 & 0.667 \\
\hline
\end{tabular}

Table 5: Relatives Preference Grades and Overall Relative Preference Grades of P2

$\mathrm{P} 2=\{\mathrm{CS} / 0.857, \mathrm{~B} / 0.833, \mathrm{~F} / 0.667, \mathrm{M} / 0.286, \mathrm{P} / 0.222\}$

\begin{tabular}{|c|c|c|c|c|c|}
\hline $\boldsymbol{f}\left(\boldsymbol{x}_{\boldsymbol{i}}, \boldsymbol{x}_{\boldsymbol{j}}\right)$ & $\mathbf{P}$ & $\mathbf{C S}$ & $\mathbf{B}$ & $\mathbf{M}$ & $\mathbf{F}$ \\
\hline $\mathrm{P}$ & 0 & 5 & 5 & 6 & 7 \\
\hline $\mathrm{CS}$ & 8 & 0 & 6 & 9 & 8 \\
\hline $\mathrm{B}$ & 6 & 5 & 0 & 7 & 7 \\
\hline $\mathrm{M}$ & 4 & 3 & 4 & 0 & 7 \\
\hline $\mathrm{F}$ & 4 & 4 & 5 & 8 & 0 \\
\hline
\end{tabular}

Table 6: The Desirability Grades Prepared by P3 


\begin{tabular}{|c|c|c|c|c|c|c|}
\hline $\boldsymbol{F}\left(\boldsymbol{x}_{\boldsymbol{i}}, \boldsymbol{x}_{\boldsymbol{j}}\right)$ & $\mathbf{P}$ & $\mathbf{C S}$ & $\mathbf{B}$ & $\mathbf{M}$ & $\mathbf{F}$ & $\boldsymbol{P}\left(\boldsymbol{x}_{\boldsymbol{i}}\right)$ \\
\hline $\mathrm{P}$ & 1.000 & 0.625 & 0.833 & 1.000 & 1.000 & 0.625 \\
\hline $\mathrm{CS}$ & 1.000 & 1.000 & 1.000 & 1.000 & 1.000 & 1.000 \\
\hline $\mathrm{B}$ & 1.000 & 0.833 & 1.000 & 1.000 & 1.000 & 0.833 \\
\hline $\mathrm{M}$ & 0.667 & 0.333 & 0.571 & 1.000 & 0.875 & 0.333 \\
\hline $\mathrm{F}$ & 0.571 & 0.500 & 0.714 & 1.000 & 1.000 & 0.500 \\
\hline
\end{tabular}

Table 7: Relatives Preference Grades and Overall Relative Preference Grades of P3

$\mathrm{P} 3=\{\mathrm{CS} / 1.000, \mathrm{~B} / 0.833, \mathrm{P} / 0.625, \mathrm{~F} / 0.500, \mathrm{M} / 0.333\}$

\begin{tabular}{|c|c|c|c|c|c|}
\hline$f\left(x_{i}, x_{j}\right)$ & $\mathrm{P}$ & $\mathrm{CS}$ & $\mathrm{B}$ & $\mathrm{M}$ & $\mathrm{F}$ \\
\hline $\mathrm{P}$ & 0 & 1 & 3 & 9 & 8 \\
\hline $\mathrm{CS}$ & 2 & 0 & 3 & 4 & 8 \\
\hline $\mathrm{B}$ & 7 & 9 & 0 & 9 & 8 \\
\hline $\mathrm{M}$ & 4 & 5 & 7 & 0 & 8 \\
\hline $\mathrm{F}$ & 5 & 6 & 7 & 7 & 0 \\
\hline
\end{tabular}

Table 8: The Desirability Grades Prepared by P4

\begin{tabular}{|c|c|c|c|c|c|c|}
\hline $\boldsymbol{F}\left(\boldsymbol{x}_{\boldsymbol{i}}, \boldsymbol{x}_{\boldsymbol{j}}\right)$ & $\mathbf{P}$ & $\mathbf{C S}$ & $\mathbf{B}$ & $\mathbf{M}$ & $\mathbf{F}$ & $\boldsymbol{P}\left(\boldsymbol{x}_{\boldsymbol{i}}\right)$ \\
\hline $\mathrm{P}$ & 1.000 & 0.500 & 0.429 & 1.000 & 1.000 & 0.429 \\
\hline $\mathrm{CS}$ & 1.000 & 1.000 & 0.333 & 0.800 & 1.000 & 0.333 \\
\hline $\mathrm{B}$ & 1.000 & 1.000 & 1.000 & 1.000 & 1.000 & 1.000 \\
\hline $\mathrm{M}$ & 0.444 & 1.000 & 0.778 & 1.000 & 1.000 & 0.444 \\
\hline F & 0.625 & 0.750 & 0.875 & 0.875 & 1.000 & 0.625 \\
\hline
\end{tabular}

Table 9: Relatives Preference Grades and Overall Relative Preference Grades of P4

$\mathrm{P} 4=\{\mathrm{B} / 1.000, \mathrm{~F} / 0.625, \mathrm{M} / 0.444, \mathrm{P} / 0.429, \mathrm{C} . \mathrm{S} / 0.333\}$

\begin{tabular}{|c|c|c|c|c|c|}
\hline $\boldsymbol{f}\left(\boldsymbol{x}_{\boldsymbol{i}}, \boldsymbol{x}_{\boldsymbol{j}}\right)$ & $\mathbf{P}$ & $\mathbf{C S}$ & $\mathbf{B}$ & $\mathbf{M}$ & $\mathbf{F}$ \\
\hline $\mathrm{P}$ & 0 & 3 & 4 & 5 & 3 \\
\hline $\mathrm{CS}$ & 6 & 0 & 4 & 6 & 7 \\
\hline $\mathrm{B}$ & 3 & 5 & 0 & 8 & 5 \\
\hline $\mathrm{M}$ & 2 & 3 & 6 & 0 & 5 \\
\hline $\mathrm{F}$ & 5 & 4 & 4 & 4 & 0 \\
\hline
\end{tabular}

Table 10: The Desirability Grades Prepared by P5

\begin{tabular}{|c|c|c|c|c|c|c|}
\hline $\boldsymbol{F}\left(\boldsymbol{x}_{\boldsymbol{i}}, \boldsymbol{x}_{\boldsymbol{j}}\right)$ & $\mathbf{P}$ & $\mathbf{C S}$ & $\mathbf{B}$ & $\mathbf{M}$ & $\mathbf{F}$ & $\boldsymbol{P}\left(\boldsymbol{x}_{\boldsymbol{i}}\right)$ \\
\hline $\mathrm{P}$ & 1.000 & 0.500 & 1.000 & 1.000 & 0.600 & 0.500 \\
\hline $\mathrm{CS}$ & 1.000 & 1.000 & 0.800 & 1.000 & 1.000 & 0.800 \\
\hline $\mathrm{B}$ & 0.750 & 1.000 & 1.000 & 1.000 & 1.000 & 0.750 \\
\hline $\mathrm{M}$ & 0.400 & 0.500 & 0.750 & 1.000 & 1.000 & 0.400 \\
\hline $\mathrm{F}$ & 1.000 & 0.571 & 0.800 & 0.800 & 1.000 & 0.571 \\
\hline
\end{tabular}

Table 11: Relatives Preference Grades and Overall Relative Preference Grades of P5

$\mathrm{P} 5=\{\mathrm{CS} / 0.800, \mathrm{~B} / 0.750, \mathrm{~F} / 0.571, \mathrm{P} / 0.500, \mathrm{M} / 0.400\}$

\begin{tabular}{|c|c|c|c|c|c|}
\hline $\boldsymbol{f}\left(\boldsymbol{x}_{\boldsymbol{i}}, \boldsymbol{x}_{\boldsymbol{j}}\right)$ & $\mathbf{P}$ & $\mathbf{C S}$ & $\mathbf{B}$ & $\mathbf{M}$ & $\mathbf{F}$ \\
\hline $\mathrm{P}$ & 0 & 8 & 5 & 7 & 4 \\
\hline $\mathrm{CS}$ & 2 & 0 & 4 & 4 & 6 \\
\hline $\mathrm{B}$ & 8 & 8 & 0 & 6 & 7 \\
\hline $\mathrm{M}$ & 3 & 5 & 4 & 0 & 4 \\
\hline $\mathrm{F}$ & 9 & 8 & 5 & 5 & 0 \\
\hline
\end{tabular}

Table 12: The Desirability Grades Prepared by P5 


\begin{tabular}{|c|c|c|c|c|c|c|}
\hline $\boldsymbol{F}\left(\boldsymbol{x}_{\boldsymbol{i}}, \boldsymbol{x}_{\boldsymbol{j}}\right)$ & $\mathbf{P}$ & $\mathbf{C S}$ & $\mathbf{B}$ & $\mathbf{M}$ & $\mathbf{F}$ & $\boldsymbol{P}\left(\boldsymbol{x}_{\boldsymbol{i}}\right)$ \\
\hline P & 1.000 & 1.000 & 0.625 & 1.000 & 0.444 & 0.444 \\
\hline CS & 0.250 & 1.000 & 0.500 & 0.800 & 0.750 & 0.250 \\
\hline B & 1.000 & 1.000 & 1.000 & 1.000 & 1.000 & 1.000 \\
\hline M & 0.429 & 0.833 & 0.667 & 1.000 & 0.800 & 0.429 \\
\hline F & 1.000 & 1.000 & 0.714 & 1.000 & 1.000 & 0.714 \\
\hline
\end{tabular}

Table 13: Relatives Preference Grades and Overall Relative Preference Grades of P5

$\mathrm{P} 6=\{\mathrm{B} / 1.000, \mathrm{~F} / 0.714, \mathrm{P} / 0.444, \mathrm{M} / 0.429, \mathrm{CS} / 0.250\}$

\begin{tabular}{|c|c|c|c|c|c|}
\hline$f\left(x_{i}, x_{j}\right)$ & $\mathrm{P}$ & $\mathrm{CS}$ & $\mathrm{B}$ & $\mathrm{M}$ & $\mathrm{F}$ \\
\hline $\mathrm{P}$ & 0 & 3 & 4 & 5 & 8 \\
\hline $\mathrm{CS}$ & 2 & 0 & 3 & 4 & 6 \\
\hline $\mathrm{B}$ & 7 & 9 & 0 & 6 & 8 \\
\hline $\mathrm{M}$ & 6 & 7 & 4 & 0 & 7 \\
\hline $\mathrm{F}$ & 5 & 7 & 3 & 6 & 0 \\
\hline
\end{tabular}

Table 14: The Desirability Grades Prepared by P7

\begin{tabular}{|c|c|c|c|c|c|c|}
\hline $\boldsymbol{F}\left(\boldsymbol{x}_{\boldsymbol{i}}, \boldsymbol{x}_{\boldsymbol{j}}\right)$ & $\mathbf{P}$ & $\mathbf{C S}$ & $\mathbf{B}$ & $\mathbf{M}$ & $\mathbf{F}$ & $\boldsymbol{P}\left(\boldsymbol{x}_{\boldsymbol{i}}\right)$ \\
\hline $\mathrm{P}$ & 1.000 & 1.000 & 0.571 & 0.833 & 1.000 & 0.571 \\
\hline $\mathrm{CS}$ & 0.667 & 1.000 & 0.333 & 0.571 & 0.857 & 0.333 \\
\hline $\mathrm{B}$ & 1.000 & 1.000 & 1.000 & 1.000 & 1.000 & 1.000 \\
\hline $\mathrm{M}$ & 1.000 & 1.000 & 0.667 & 1.000 & 1.000 & 0.667 \\
\hline $\mathrm{F}$ & 0.625 & 1.000 & 0.375 & 0.857 & 1.000 & 0.375 \\
\hline
\end{tabular}

Table 15: Relatives Preference Grades and Overall Relative Preference Grades of P7

$\mathrm{P} 7=\{\mathrm{B} / 1.000, \mathrm{M} / 0.667, \mathrm{P} / 0.571, \mathrm{~F} / 0.375, \mathrm{CS} / 0.333\}$

\begin{tabular}{|c|c|c|c|c|c|}
\hline $\boldsymbol{f}\left(\boldsymbol{x}_{\boldsymbol{i}}, \boldsymbol{x}_{\boldsymbol{j}}\right)$ & $\mathbf{P}$ & $\mathbf{C S}$ & $\mathbf{B}$ & $\mathbf{M}$ & $\mathbf{F}$ \\
\hline $\mathrm{P}$ & 0 & 2 & 1 & 5 & 4 \\
\hline $\mathrm{CS}$ & 4 & 0 & 1 & 3 & 3 \\
\hline $\mathrm{B}$ & 5 & 2 & 0 & 3 & 3 \\
\hline $\mathrm{M}$ & 4 & 2 & 1 & 0 & 3 \\
\hline $\mathrm{F}$ & 3 & 4 & 2 & 5 & 0 \\
\hline
\end{tabular}

Table 16: The Desirability Grades Prepared By P8

\begin{tabular}{|c|c|c|c|c|c|c|}
\hline $\boldsymbol{F}\left(\boldsymbol{x}_{\boldsymbol{i}}, \boldsymbol{x}_{\boldsymbol{j}}\right)$ & $\mathbf{P}$ & $\mathbf{C S}$ & $\mathbf{B}$ & $\mathbf{M}$ & $\mathbf{F}$ & $\boldsymbol{P}\left(\boldsymbol{x}_{\boldsymbol{i}}\right)$ \\
\hline $\mathrm{P}$ & 1.000 & 0.500 & 0.200 & 1.000 & 1.000 & 0.200 \\
\hline $\mathrm{CS}$ & 1.000 & 1.000 & 0.500 & 1.000 & 0.750 & 0.500 \\
\hline $\mathrm{B}$ & 1.000 & 1.000 & 1.000 & 1.000 & 1.000 & 1.000 \\
\hline $\mathrm{M}$ & 0.800 & 0.667 & 0.333 & 1.000 & 0.600 & 0.333 \\
\hline $\mathrm{F}$ & 0.750 & 1.000 & 0.667 & 1.000 & 1.000 & 0.667 \\
\hline
\end{tabular}

Table 17: Relatives Preference Grades and Overall Relative Preference Grades of P8

$P 8=\{B / 1.000, F / 0.667, C S / 0.500, M / 0.333, P / 0.200\}$

\begin{tabular}{|c|c|c|c|c|c|}
\hline $\boldsymbol{f}\left(\boldsymbol{x}_{\boldsymbol{i}}, \boldsymbol{x}_{\boldsymbol{j}}\right)$ & $\mathbf{P}$ & $\mathbf{C S}$ & $\mathbf{B}$ & $\mathbf{M}$ & $\mathbf{F}$ \\
\hline $\mathrm{P}$ & 0 & 8 & 5 & 7 & 4 \\
\hline $\mathrm{CS}$ & 1 & 0 & 4 & 5 & 5 \\
\hline $\mathrm{B}$ & 8 & 8 & 0 & 6 & 7 \\
\hline $\mathrm{M}$ & 3 & 5 & 4 & 0 & 5 \\
\hline F & 9 & 8 & 5 & 5 & 0 \\
\hline
\end{tabular}

Table 18: The Desirability Grades Prepared by $P 9$ 


\begin{tabular}{|c|c|c|c|c|c|c|}
\hline $\boldsymbol{F}\left(\boldsymbol{x}_{\boldsymbol{i}}, \boldsymbol{x}_{\boldsymbol{j}}\right)$ & $\mathbf{P}$ & $\mathbf{C S}$ & $\mathbf{B}$ & $\mathbf{M}$ & $\mathbf{F}$ & $\boldsymbol{P}\left(\boldsymbol{x}_{\boldsymbol{i}}\right)$ \\
\hline $\mathrm{P}$ & 1.000 & 1.000 & 0.625 & 1.000 & 0.444 & 0.444 \\
\hline $\mathrm{CS}$ & 0.125 & 1.000 & 0.500 & 1.000 & 0.625 & 0.125 \\
\hline $\mathrm{B}$ & 1.000 & 1.000 & 1.000 & 1.000 & 1.000 & 1.000 \\
\hline $\mathrm{M}$ & 0.429 & 1.000 & 0.667 & 1.000 & 1.000 & 0.429 \\
\hline $\mathrm{F}$ & 1.000 & 1.000 & 0.714 & 1.000 & 1.000 & 0.714 \\
\hline
\end{tabular}

Table 19: Relatives Preference Grades and Overall Relative Preference Grades of $P 9$

$\mathrm{P} 9=\{\mathrm{B} / 1.000, \mathrm{~F} / 0.714, \mathrm{P} / 0.444, \mathrm{M} / 0.429, \mathrm{CS} / 0.125\}$

\begin{tabular}{|c|c|c|c|c|c|}
\hline $\boldsymbol{f}\left(\boldsymbol{x}_{\boldsymbol{i}}, \boldsymbol{x}_{\boldsymbol{j}}\right)$ & $\mathbf{P}$ & $\mathbf{C S}$ & $\mathbf{B}$ & $\mathbf{M}$ & $\mathbf{F}$ \\
\hline $\mathrm{P}$ & 0 & 1 & 3 & 4 & 2 \\
\hline $\mathrm{CS}$ & 7 & 0 & 9 & 5 & 4 \\
\hline $\mathrm{B}$ & 9 & 7 & 0 & 5 & 5 \\
\hline $\mathrm{M}$ & 8 & 2 & 4 & 0 & 4 \\
\hline $\mathrm{F}$ & 7 & 6 & 7 & 5 & 0 \\
\hline
\end{tabular}

Table 20: The Desirability Grades Prepared By P10

\begin{tabular}{|c|c|c|c|c|c|c|}
\hline $\boldsymbol{F}\left(\boldsymbol{x}_{\boldsymbol{i}}, \boldsymbol{x}_{\boldsymbol{j}}\right)$ & $\mathbf{P}$ & $\mathbf{C S}$ & $\mathbf{B}$ & $\mathbf{M}$ & $\mathbf{F}$ & $\boldsymbol{P}\left(\boldsymbol{x}_{\boldsymbol{i}}\right)$ \\
\hline $\mathrm{P}$ & 1.000 & 0.143 & 0.333 & 0.500 & 0.286 & 0.143 \\
\hline $\mathrm{CS}$ & 1.000 & 1.000 & 1.000 & 1.000 & 0.667 & 0.667 \\
\hline B & 1.000 & 0.778 & 1.000 & 1.000 & 0.714 & 0.714 \\
\hline M & 1.000 & 0.400 & 0.800 & 1.000 & 0.800 & 0.400 \\
\hline F & 1.000 & 1.000 & 1.000 & 1.000 & 1.000 & 1.000 \\
\hline
\end{tabular}

Table 21: Relatives Preference Grades and Overall Relative Preference Grades Of P10

$\mathrm{P}_{10}=\{\mathrm{F} / 1.000, \mathrm{~B} / 0.714, \mathrm{CS} / 0.667, \mathrm{M} / 0.400, \mathrm{P} / 0.143\}$

For simplicity, let $P=a_{1}, C S=a_{2}, B=a_{3}, M=a_{4}$ and $F=a_{5}$,

Then, by this approach the following fuzzy sets as the preference ordering of all the ten graduates were obtained:

$\mathrm{P}_{1}=\left\{a_{2}, a_{5}, a_{3}, a_{4}, a_{1}\right\}, \mathrm{P}_{2}=\left\{a_{2}, a_{3}, a_{5}, a_{4}, a_{1}\right\}, \mathrm{P}_{3}=\left\{a_{2}, a_{3}, a_{1}, a_{5}, a_{4}\right\}, \mathrm{P}_{4}=\left\{a_{3}, a_{5}, a_{4}, a_{1}, a_{2}\right\}, \mathrm{P}_{5}=\left\{a_{2}, a_{3}, a_{5}, a_{1}, a_{4}\right\}$,

$\mathrm{P}_{6}=\left\{a_{3}, a_{5}, a_{1}, a_{4}, a_{2}\right\}, \mathrm{P}_{7}=\left\{a_{3}, a_{4}, a_{1}, a_{5}, a_{2}\right\}, \mathrm{P}_{8}=\left\{a_{3}, a_{5}, a_{2}, a_{4}, a_{1}\right\}, \mathrm{P}_{9}=\left\{a_{3}, a_{5}, a_{1}, a_{4}, a_{2}\right\}, \mathrm{P}_{10}=\left\{a_{5}, a_{3}, a_{2}, a_{4}, a_{1}\right\}$

\section{Calculating the Overall Optimal Preference Ordering (First Set)}

Having the ten ordered sets above, Blin approach is applied for the calculation of the overall optimal preference ordering for the group. Using the membership function given in (3) for fuzzy group preferences ordering relation $S$ (where $n=10$ ), the following fuzzy social preferences relation is arrived at:

\begin{tabular}{|c|c|c|c|c|}
\hline$a_{1}$ & $a_{2}$ & $a_{3}$ & $a_{4}$ & $a_{5}$ \\
\hline$[0.0$ & 0.4 & 0.0 & 0.4 & 0.2 \\
\hline 0.6 & 0.0 & 0.4 & 0.6 & 0.4 \\
\hline 1.0 & 0.6 & 0.0 & 1.0 & 0.8 \\
\hline 0.6 & 0.4 & 0.0 & 0.0 & 0.1 \\
\hline 0.8 & 0.6 & 0.2 & 0.9 & 0.0 \\
\hline
\end{tabular}

The $\alpha$-cuts of this fuzzy relation $S$ are:

$$
\begin{gathered}
{ }^{1} S=\left\{\left(a_{3}, a_{1}\right),\left(a_{3}, a_{4}\right)\right\},{ }^{0.9} S=\left\{{ }^{1} S,\left(a_{5}, a_{4}\right)\right\},{ }^{0.8} S=\left\{{ }^{0.9} S,\left(a_{3}, a_{5}\right),\left(a_{5}, a_{1}\right)\right\} \\
{ }^{0.6} S=\left\{{ }^{0.8} S,\left(a_{2}, a_{1}\right),\left(a_{2}, a_{4}\right),\left(a_{3}, a_{2}\right),\left(a_{4}, a_{1}\right),\left(a_{5}, a_{2}\right)\right\},{ }^{0.4} S=\left\{{ }^{0.6} S,\left(a_{1}, a_{2}\right),\left(a_{1}, a_{4}\right),\left(a_{2}, a_{3}\right),\left(a_{2}, a_{4}\right),\left(a_{4}, a_{2}\right)\right\} \\
{ }^{0.2} S=\left\{{ }^{0.4} S,\left(a_{1}, a_{5}\right),\left(a_{5}, a_{3}\right)\right\} \text { and }{ }^{0.1} S=\left\{{ }^{0.1} S,\left(a_{4}, a_{5}\right)\right\}
\end{gathered}
$$

Where $\left(a_{i}, a_{j}\right)$ means $a_{i}$ precedes $a_{j}$. Then, the intersection of the classes of crisp total ordering that are compatible with the pairs in the $\alpha$-cuts ${ }^{\alpha} S$ in order to arrive at unique crisp ordering that constitutes the group choice is taken. 
The total orderings, ${ }^{T} O$, on $\mathrm{X} \times \mathrm{X}$, which is the set of all the five-elements sets that can be generated from $X=$ $\left\{a_{1}, a_{2}, a_{3}, a_{4}, a_{5}\right\}$, which by permutation are 120 different ordered sets, are obtained and 40 out of those 120 sets are compatible with the set of ${ }^{1} S$ and they are:

$$
\begin{gathered}
{ }^{1} O=\left\{\left\{a_{5}, a_{3}, a_{4}, a_{1}, a_{2}\right\},\left\{a_{5}, a_{3}, a_{4}, a_{2}, a_{1}\right\},\left\{a_{5}, a_{3}, a_{1}, a_{4}, a_{2}\right\},\left\{a_{5}, a_{3}, a_{1}, a_{2}, a_{4}\right\},\left\{a_{5}, a_{3}, a_{2}, a_{1}, a_{4}\right\},\left\{a_{5}, a_{3}, a_{2}, a_{4}, a_{1}\right\}\right. \\
\left\{a_{5}, a_{2}, a_{3}, a_{1}, a_{4}\right\},\left\{a_{5}, a_{2}, a_{3}, a_{4}, a_{1}\right\},\left\{a_{3}, a_{4}, a_{5}, a_{1}, a_{2}\right\},\left\{a_{3}, a_{4}, a_{5}, a_{2}, a_{1}\right\},\left\{a_{3}, a_{4}, a_{1}, a_{5}, a_{2}\right\},\left\{a_{3}, a_{4}, a_{1}, a_{2}, a_{5}\right\} \\
\left\{a_{3}, a_{4}, a_{2}, a_{1}, a_{5}\right\},\left\{a_{3}, a_{4}, a_{2}, a_{5}, a_{1}\right\},\left\{a_{3}, a_{5}, a_{4}, a_{1}, a_{2}\right\},\left\{a_{3}, a_{5}, a_{4}, a_{2}, a_{1}\right\},\left\{a_{3}, a_{5}, a_{1}, a_{4}, a_{2}\right\},\left\{a_{3}, a_{5}, a_{1}, a_{2}, a_{4}\right\} \\
\left\{a_{3}, a_{5}, a_{2}, a_{1}, a_{4}\right\},\left\{a_{3}, a_{5}, a_{2}, a_{4}, a_{1}\right\},\left\{a_{3}, a_{1}, a_{5}, a_{4}, a_{2}\right\},\left\{a_{3}, a_{1}, a_{5}, a_{2}, a_{4}\right\},\left\{a_{3}, a_{1}, a_{4}, a_{5}, a_{2}\right\},\left\{a_{3}, a_{1}, a_{4}, a_{2}, a_{5}\right\} \\
\left\{a_{3}, a_{1}, a_{2}, a_{4}, a_{5}\right\},\left\{a_{3}, a_{1}, a_{2}, a_{5}, a_{4}\right\},\left\{a_{3}, a_{2}, a_{5}, a_{1}, a_{4}\right\},\left\{a_{3}, a_{2}, a_{5}, a_{4}, a_{1}\right\},\left\{a_{3}, a_{2}, a_{1}, a_{5}, a_{4}\right\},\left\{a_{3}, a_{2}, a_{1}, a_{4}, a_{5}\right\} \\
\left\{a_{3}, a_{2}, a_{4}, a_{1}, a_{5}\right\},\left\{a_{3}, a_{2}, a_{4}, a_{5}, a_{1}\right\},\left\{a_{2}, a_{3}, a_{4}, a_{1}, a_{5}\right\},\left\{a_{2}, a_{3}, a_{4}, a_{5}, a_{1}\right\},\left\{a_{2}, a_{3}, a_{1}, a_{4}, a_{5}\right\},\left\{a_{2}, a_{3}, a_{1}, a_{5}, a_{4}\right\} \\
\left.\left\{a_{2}, a_{3}, a_{5}, a_{1}, a_{4}\right\},\left\{a_{2}, a_{3}, a_{5}, a_{4}, a_{1}\right\},\left\{a_{2}, a_{5}, a_{3}, a_{1}, a_{4}\right\},\left\{a_{2}, a_{5}, a_{3}, a_{4}, a_{1}\right\}\right\}
\end{gathered}
$$

Thus, ${ }^{T} O \cap{ }^{1} O={ }^{1} O$

The total orderings ${ }^{0.9} \mathrm{O}$ that are compatible with the pairs in the crisp relation ${ }^{0.9} \mathrm{~S}$ are 25 out of the above 40 sets, that is:

$$
\begin{aligned}
{ }^{0.9} O= & \left\{\left\{a_{5}, a_{3}, a_{4}, a_{1}, a_{2}\right\},\left\{a_{5}, a_{3}, a_{4}, a_{2}, a_{1}\right\},\left\{a_{5}, a_{3}, a_{1}, a_{4}, a_{2}\right\},\left\{a_{5}, a_{3}, a_{1}, a_{2}, a_{4}\right\},\left\{a_{5}, a_{3}, a_{2}, a_{1}, a_{4}\right\},\left\{a_{5}, a_{3}, a_{2}, a_{4}, a_{1}\right\}\right. \\
& \left\{a_{5}, a_{2}, a_{3}, a_{1}, a_{4}\right\},\left\{a_{5}, a_{2}, a_{3}, a_{4}, a_{1}\right\},\left\{a_{3}, a_{5}, a_{4}, a_{1}, a_{2}\right\},\left\{a_{3}, a_{5}, a_{4}, a_{2}, a_{1}\right\},\left\{a_{3}, a_{5}, a_{1}, a_{4}, a_{2}\right\},\left\{a_{3}, a_{5}, a_{1}, a_{2}, a_{4}\right\}, \\
& \left\{a_{3}, a_{5}, a_{2}, a_{1}, a_{4}\right\},\left\{a_{3}, a_{5}, a_{2}, a_{4}, a_{1}\right\},\left\{a_{3}, a_{1}, a_{5}, a_{4}, a_{2}\right\},\left\{a_{3}, a_{1}, a_{5}, a_{2}, a_{4}\right\},\left\{a_{3}, a_{1}, a_{2}, a_{5}, a_{4}\right\},\left\{a_{3}, a_{2}, a_{5}, a_{1}, a_{4}\right\}, \\
& \left\{a_{3}, a_{2}, a_{5}, a_{4}, a_{1}\right\},\left\{a_{3}, a_{2}, a_{1}, a_{5}, a_{4}\right\},\left\{a_{2}, a_{3}, a_{1}, a_{5}, a_{4}\right\},\left\{a_{2}, a_{3}, a_{5}, a_{1}, a_{4}\right\},\left\{a_{2}, a_{3}, a_{5}, a_{4}, a_{1}\right\},\left\{a_{2}, a_{5}, a_{3}, a_{1}, a_{4}\right\},
\end{aligned}
$$

$$
\left.\left\{a_{2}, a_{5}, a_{3}, a_{4}, a_{1}\right\}\right\}
$$

Thus, ${ }^{1} \mathrm{O} \cap{ }^{0.9} \mathrm{O}={ }^{0.9} \mathrm{O}$

The total orderings ${ }^{0.8} O$ that are compatible with the pairs in the crisp relation ${ }^{0.8} S$ are 12 out of the above 25 sets, that is:

$$
\begin{gathered}
{ }^{0.8} O=\left\{\left\{a_{3}, a_{5}, a_{4}, a_{1}, a_{2}\right\},\left\{a_{3}, a_{5}, a_{4}, a_{2}, a_{1}\right\},\left\{a_{3}, a_{5}, a_{1}, a_{4}, a_{2}\right\},\left\{a_{3}, a_{5}, a_{1}, a_{2}, a_{4}\right\},\left\{a_{3}, a_{5}, a_{2}, a_{1}, a_{4}\right\},\left\{a_{3}, a_{5}, a_{2}, a_{4}, a_{1}\right\}\right. \\
\left.\left\{a_{3}, a_{2}, a_{5}, a_{1}, a_{4}\right\},\left\{a_{3}, a_{2}, a_{5}, a_{4}, a_{1}\right\},\left\{a_{2}, a_{3}, a_{5}, a_{1}, a_{4}\right\},\left\{a_{2}, a_{3}, a_{5}, a_{4}, a_{1}\right\},\left\{a_{2}, a_{5}, a_{3}, a_{1}, a_{4}\right\},\left\{a_{2}, a_{5}, a_{3}, a_{4}, a_{1}\right\}\right\}
\end{gathered}
$$

Thus, ${ }^{0.9} \mathrm{O} \cap{ }^{0.8} \mathrm{O}={ }^{0.8} \mathrm{O}$

The total orderings ${ }^{0.6} \mathrm{O}$ that are compatible with the pairs in the crisp relation ${ }^{0.6} \mathrm{~S}$ is 1 out of the above 12 sets, that is:

$$
{ }^{0.6} \mathrm{O}=\left\{\left\{a_{3}, a_{5}, a_{2}, a_{4}, a_{1}\right\}\right\}
$$

finally, ${ }^{1} O \cap{ }^{0.9} O \cap{ }^{0.8} O^{0.6} O={ }^{0.6} O=\left\{\left\{a_{3}, a_{5}, a_{2}, a_{4}, a_{1}\right\}\right\}$

\section{Ordering Based on Direct Preferencing}

The second phase of this work is by direct preferencing on the same alternatives and by the same group of people. By this approach the preference orderings of all the ten graduates were obtained and the corresponding sets were represented by $\mathrm{P}_{\mathrm{k}}^{*}, k \in N$ as below:

$\mathrm{P}_{1}^{*}=\left\{a_{2}, a_{5}, a_{3}, a_{4}, a_{1}\right\}, \mathrm{P}_{2}^{*}=\left\{a_{2}, a_{3}, a_{4}, a_{1}, a_{5}\right\}, \mathrm{P}_{3}^{*}=\left\{a_{2}, a_{3}, a_{1}, a_{5}, a_{4}\right\}, \mathrm{P}_{4}^{*}=\left\{a_{3}, a_{5}, a_{1}, a_{2}, a_{4}\right\}, \mathrm{P}_{5}^{*}=\left\{a_{5}, a_{3}, a_{4}, a_{1}, a_{2}\right\}$,

$\mathrm{P}_{6}^{*}=\left\{a_{3}, a_{5}, a_{2}, a_{4}, a_{1}\right\}, \mathrm{P}_{7}^{*}=\left\{a_{3}, a_{4}, a_{1}, a_{5}, a_{2}\right\}, \mathrm{P}_{8}^{*}=\left\{a_{3}, a_{2}, a_{5}, a_{4}, a_{1}\right\}, \mathrm{P}_{9}^{*}=\left\{a_{2}, a_{1}, a_{3}, a_{5}, a_{4}\right\}, \mathrm{P}_{10}^{*}=\left\{a_{3}, a_{2}, a_{4}, a_{5}, a_{1}\right\}$

\section{Calculating the Overall Optimal Preference Ordering (Second Set)}

Having the ten ordered sets above, Blin approach is applied, also, for the calculation of the overall optimal preference ordering for the group. Similarly, using the membership function given in (3) for fuzzy group preferences ordering relation $S$ (where $n=10$ ), the following fuzzy social preferences relation is obtained: 


\begin{tabular}{|c|c|c|c|c|c|}
\hline & $a_{1}$ & $a_{2}$ & $a_{3}$ & $a_{4}$ & $a_{5}$ \\
\hline$a_{1}$ & {$[0.0$} & 0.3 & 0.1 & 0.3 & 0.4 \\
\hline$a_{2}$ & 0.7 & 0.0 & 0.4 & 0.8 & 0.6 \\
\hline$a_{3}$ & 0.9 & 0.6 & 0.0 & 1.0 & 0.8 \\
\hline$a_{4}$ & 0.7 & 0.2 & 0.0 & 0.0 & 0.3 \\
\hline$a_{5}$ & $L_{0.6}$ & 0.4 & 0.2 & 0.7 & $0,0]$ \\
\hline
\end{tabular}

The $\alpha$-cuts of this fuzzy relation $S$ are:

$$
\begin{gathered}
{ }^{1} S=\left\{\left(a_{3}, a_{4}\right)\right\},{ }^{0.9} S=\left\{{ }^{1} S,\left(a_{3}, a_{1}\right)\right\},{ }^{0.8} S=\left\{{ }^{0.9} S,\left(a_{2}, a_{4}\right),\left(a_{3}, a_{5}\right)\right\},{ }^{0.7} S=\left\{{ }^{0.8} S,\left(a_{2}, a_{1}\right),\left(a_{4}, a_{1}\right),\left(a_{5}, a_{4}\right)\right\}, \\
{ }^{0.6} S=\left\{{ }^{0.7} S,\left(a_{2}, a_{5}\right),\left(a_{3}, a_{2}\right),\left(a_{5}, a_{1}\right)\right\},{ }^{0.4} S=\left\{{ }^{0.6} S,\left(a_{1}, a_{5}\right),\left(a_{2}, a_{3}\right),\left(a_{5}, a_{2}\right)\right\},{ }^{0.3} S=\left\{{ }^{0.4} S,\left(a_{1}, a_{2}\right),\left(a_{1}, a_{4}\right),\left(a_{4}, a_{5}\right)\right\}, \\
{ }^{0.2} S=\left\{{ }^{0.3} S,\left(a_{4}, a_{2}\right),\left(a_{5}, a_{3}\right)\right\},{ }^{0.1} S=\left\{{ }^{0.2} S,\left(a_{1}, a_{3}\right)\right\} \text { and }{ }^{0} S=\left\{0.1 S,\left(a_{4}, a_{3}\right)\right\}
\end{gathered}
$$

Where $\left(a_{i}, a_{j}\right)$ means $a_{i}$ precedes $a_{j}$. Then, the intersection of the classes of crisp total ordering that are compatible with the pairs in the $\alpha$-cuts ${ }^{\alpha} S$ in order to arrive at unique crisp ordering that constitutes the group choice is taken as well.

The total ordering, ${ }^{T} O$, on $\mathrm{X} \times \mathrm{X}$, over $X=\left\{a_{1}, a_{2}, a_{3}, a_{4}, a_{5}\right\}$, were obtained and 60 out of the 120 sets are compatible with the set of ${ }^{1} S$ and they are:

$$
\begin{aligned}
& { }^{1} O=\left\{\left\{a_{5}, a_{3}, a_{4}, a_{1}, a_{2}\right\},\left\{a_{5}, a_{3}, a_{4}, a_{2}, a_{1}\right\},\left\{a_{5}, a_{3}, a_{1}, a_{4}, a_{2}\right\},\left\{a_{5}, a_{3}, a_{1}, a_{2}, a_{4}\right\},\left\{a_{5}, a_{3}, a_{2}, a_{1}, a_{4}\right\},\left\{a_{5}, a_{3}, a_{2}, a_{4}, a_{1}\right\},\right. \\
& \left\{a_{5}, a_{1}, a_{3}, a_{4}, a_{2}\right\},\left\{a_{5}, a_{1}, a_{3}, a_{2}, a_{4}\right\},\left\{a_{5}, a_{1}, a_{2}, a_{3}, a_{4}\right\}\left\{a_{5}, a_{2}, a_{3}, a_{1}, a_{4}\right\},\left\{a_{5}, a_{2}, a_{3}, a_{4}, a_{1}\right\},\left\{a_{5}, a_{2}, a_{1}, a_{3}, a_{4}\right\} \\
& \left\{a_{3}, a_{4}, a_{5}, a_{1}, a_{2}\right\},\left\{a_{3}, a_{4}, a_{5}, a_{2}, a_{1}\right\},\left\{a_{3}, a_{4}, a_{1}, a_{5}, a_{2}\right\},\left\{a_{3}, a_{4}, a_{1}, a_{2}, a_{5}\right\},\left\{a_{3}, a_{4}, a_{2}, a_{1}, a_{5}\right\},\left\{a_{3}, a_{4}, a_{2}, a_{5}, a_{1}\right\} \text {, } \\
& \left\{a_{3}, a_{5}, a_{4}, a_{1}, a_{2}\right\},\left\{a_{3}, a_{5}, a_{4}, a_{2}, a_{1}\right\},\left\{a_{3}, a_{5}, a_{1}, a_{4}, a_{2}\right\},\left\{a_{3}, a_{5}, a_{1}, a_{2}, a_{4}\right\},\left\{a_{3}, a_{5}, a_{2}, a_{1}, a_{4}\right\},\left\{a_{3}, a_{5}, a_{2}, a_{4}, a_{1}\right\} \text {, } \\
& \left\{a_{3}, a_{1}, a_{5}, a_{4}, a_{2}\right\},\left\{a_{3}, a_{1}, a_{5}, a_{2}, a_{4}\right\},\left\{a_{3}, a_{1}, a_{4}, a_{5}, a_{2}\right\},\left\{a_{3}, a_{1}, a_{4}, a_{2}, a_{5}\right\},\left\{a_{3}, a_{1}, a_{2}, a_{4}, a_{5}\right\},\left\{a_{3}, a_{1}, a_{2}, a_{5}, a_{4}\right\} \text {, } \\
& \left\{a_{3}, a_{2}, a_{5}, a_{1}, a_{4}\right\},\left\{a_{3}, a_{2}, a_{5}, a_{4}, a_{1}\right\},\left\{a_{3}, a_{2}, a_{1}, a_{5}, a_{4}\right\},\left\{a_{3}, a_{2}, a_{1}, a_{4}, a_{5}\right\},\left\{a_{3}, a_{2}, a_{4}, a_{1}, a_{5}\right\},\left\{a_{3}, a_{2}, a_{4}, a_{5}, a_{1}\right\} \text {, } \\
& \left\{a_{2}, a_{3}, a_{4}, a_{1}, a_{5}\right\},\left\{a_{2}, a_{3}, a_{4}, a_{5}, a_{1}\right\},\left\{a_{2}, a_{3}, a_{1}, a_{4}, a_{5}\right\},\left\{a_{2}, a_{3}, a_{1}, a_{5}, a_{4}\right\},\left\{a_{2}, a_{3}, a_{5}, a_{1}, a_{4}\right\},\left\{a_{2}, a_{3}, a_{5}, a_{4}, a_{1}\right\} \text {, } \\
& \left\{a_{2}, a_{1}, a_{3}, a_{4}, a_{5}\right\},\left\{a_{2}, a_{1}, a_{3}, a_{5}, a_{4}\right\},\left\{a_{2}, a_{1}, a_{5}, a_{3}, a_{4}\right\},\left\{a_{2}, a_{5}, a_{3}, a_{1}, a_{4}\right\},\left\{a_{2}, a_{5}, a_{3}, a_{4}, a_{1}\right\},\left\{a_{2}, a_{5}, a_{1}, a_{3}, a_{4}\right\}, \\
& \left\{a_{1}, a_{3}, a_{4}, a_{5}, a_{2}\right\},\left\{a_{1}, a_{3}, a_{4}, a_{2}, a_{5}\right\},\left\{a_{1}, a_{3}, a_{5}, a_{4}, a_{2}\right\},\left\{a_{1}, a_{3}, a_{5}, a_{2}, a_{4}\right\},\left\{a_{1}, a_{3}, a_{2}, a_{5}, a_{4}\right\},\left\{a_{1}, a_{3}, a_{2}, a_{4}, a_{5}\right\} \text {, } \\
& \left.\left\{a_{1}, a_{5}, a_{3}, a_{4}, a_{2}\right\},\left\{a_{1}, a_{5}, a_{3}, a_{2}, a_{4}\right\},\left\{a_{1}, a_{5}, a_{2}, a_{3}, a_{4}\right\},\left\{a_{1}, a_{2}, a_{3}, a_{5}, a_{4}\right\},\left\{a_{1}, a_{2}, a_{3}, a_{4}, a_{5}\right\},\left\{a_{1}, a_{2}, a_{5}, a_{3}, a_{4}\right\}\right\}
\end{aligned}
$$

Thus, ${ }^{T} O \cap{ }^{1} O={ }^{1} O$

The total orderings ${ }^{0.9} \mathrm{O}$ that are compatible with the pairs in the crisp relation ${ }^{0.9} \mathrm{~S}$ are 40 out of the above 60 sets, that is:

$$
\begin{gathered}
{ }^{0.9} O=\left\{\left\{a_{5}, a_{3}, a_{4}, a_{1}, a_{2}\right\},\left\{a_{5}, a_{3}, a_{4}, a_{2}, a_{1}\right\},\left\{a_{5}, a_{3}, a_{1}, a_{4}, a_{2}\right\},\left\{a_{5}, a_{3}, a_{1}, a_{2}, a_{4}\right\},\left\{a_{5}, a_{3}, a_{2}, a_{1}, a_{4}\right\},\left\{a_{5}, a_{3}, a_{2}, a_{4}, a_{1}\right\},\right. \\
\left\{a_{5}, a_{2}, a_{3}, a_{1}, a_{4}\right\},\left\{a_{5}, a_{2}, a_{3}, a_{4}, a_{1}\right\},\left\{a_{3}, a_{4}, a_{5}, a_{1}, a_{2}\right\},\left\{a_{3}, a_{4}, a_{5}, a_{2}, a_{1}\right\},\left\{a_{3}, a_{4}, a_{1}, a_{5}, a_{2}\right\},\left\{a_{3}, a_{4}, a_{1}, a_{2}, a_{5}\right\} \\
\left\{a_{3}, a_{4}, a_{2}, a_{1}, a_{5}\right\},\left\{a_{3}, a_{4}, a_{2}, a_{5}, a_{1}\right\},\left\{a_{3}, a_{5}, a_{4}, a_{1}, a_{2}\right\},\left\{a_{3}, a_{5}, a_{4}, a_{2}, a_{1}\right\},\left\{a_{3}, a_{5}, a_{1}, a_{4}, a_{2}\right\},\left\{a_{3}, a_{5}, a_{1}, a_{2}, a_{4}\right\}, \\
\left\{a_{3}, a_{5}, a_{2}, a_{1}, a_{4}\right\},\left\{a_{3}, a_{5}, a_{2}, a_{4}, a_{1}\right\},\left\{a_{3}, a_{1}, a_{5}, a_{4}, a_{2}\right\},\left\{a_{3}, a_{1}, a_{5}, a_{2}, a_{4}\right\},\left\{a_{3}, a_{1}, a_{4}, a_{5}, a_{2}\right\},\left\{a_{3}, a_{1}, a_{4}, a_{2}, a_{5}\right\}, \\
\left\{a_{3}, a_{1}, a_{2}, a_{4}, a_{5}\right\},\left\{a_{3}, a_{1}, a_{2}, a_{5}, a_{4}\right\},\left\{a_{3}, a_{2}, a_{5}, a_{1}, a_{4}\right\},\left\{a_{3}, a_{2}, a_{5}, a_{4}, a_{1}\right\},\left\{a_{3}, a_{2}, a_{1}, a_{5}, a_{4}\right\},\left\{a_{3}, a_{2}, a_{1}, a_{4}, a_{5}\right\}, \\
\left\{a_{3}, a_{2}, a_{4}, a_{1}, a_{5}\right\},\left\{a_{3}, a_{2}, a_{4}, a_{5}, a_{1}\right\},\left\{a_{2}, a_{3}, a_{4}, a_{1}, a_{5}\right\},\left\{a_{2}, a_{3}, a_{4}, a_{5}, a_{1}\right\},\left\{a_{2}, a_{3}, a_{1}, a_{4}, a_{5}\right\},\left\{a_{2}, a_{3}, a_{1}, a_{5}, a_{4}\right\} \\
\left.\left\{a_{2}, a_{3}, a_{5}, a_{1}, a_{4}\right\},\left\{a_{2}, a_{3}, a_{5}, a_{4}, a_{1}\right\},\left\{a_{2}, a_{5}, a_{3}, a_{1}, a_{4}\right\},\left\{a_{2}, a_{5}, a_{3}, a_{4}, a_{1}\right\}\right\}
\end{gathered}
$$

Thus, ${ }^{1} \mathrm{O} \cap{ }^{0.9} \mathrm{O}={ }^{0.9} \mathrm{O}$

The total orderings ${ }^{0.8} \mathrm{O}$ that are compatible with the pairs in the crisp relation ${ }^{0.8} S$ are 20 out of the above 40 sets, that is:

$$
{ }^{0.8} O=\left\{\left\{a_{3}, a_{5}, a_{1}, a_{2}, a_{4}\right\},\left\{a_{3}, a_{5}, a_{2}, a_{1}, a_{4}\right\},\left\{a_{3}, a_{5}, a_{2}, a_{4}, a_{1}\right\},\left\{a_{3}, a_{1}, a_{5}, a_{2}, a_{4}\right\},\left\{a_{3}, a_{1}, a_{2}, a_{4}, a_{5}\right\},\left\{a_{3}, a_{1}, a_{2}, a_{5}, a_{4}\right\},\right.
$$




$$
\begin{gathered}
\left\{a_{3}, a_{2}, a_{5}, a_{1}, a_{4}\right\},\left\{a_{3}, a_{2}, a_{5}, a_{4}, a_{1}\right\},\left\{a_{3}, a_{2}, a_{1}, a_{5}, a_{4}\right\},\left\{a_{3}, a_{2}, a_{1}, a_{4}, a_{5}\right\},\left\{a_{3}, a_{2}, a_{4}, a_{1}, a_{5}\right\},\left\{a_{3}, a_{2}, a_{4}, a_{5}, a_{1}\right\}, \\
\left\{a_{2}, a_{3}, a_{4}, a_{1}, a_{5}\right\},\left\{a_{2}, a_{3}, a_{4}, a_{5}, a_{1}\right\},\left\{a_{2}, a_{3}, a_{1}, a_{4}, a_{5}\right\},\left\{a_{2}, a_{3}, a_{1}, a_{5}, a_{4}\right\},\left\{a_{2}, a_{3}, a_{5}, a_{1}, a_{4}\right\},\left\{a_{2}, a_{3}, a_{5}, a_{4}, a_{1}\right\} \\
\left.\left\{a_{2}, a_{5}, a_{3}, a_{1}, a_{4}\right\},\left\{a_{2}, a_{5}, a_{3}, a_{4}, a_{1}\right\}\right\}
\end{gathered}
$$

Thus, ${ }^{0.9} \mathrm{O} \cap{ }^{0.8} \mathrm{O}={ }^{0.8} \mathrm{O}$

The total orderings ${ }^{0.7} \mathrm{O}$ that are compatible with the pairs in the crisp relation ${ }^{0.7} S$ is 4 out of the above 20 sets, that is:

$$
{ }^{0.7} O=\left\{\left\{a_{3}, a_{5}, a_{2}, a_{4}, a_{1}\right\},\left\{a_{3}, a_{2}, a_{5}, a_{4}, a_{1}\right\},\left\{a_{2}, a_{3}, a_{5}, a_{4}, a_{1}\right\},\left\{a_{2}, a_{5}, a_{3}, a_{4}, a_{1}\right\}\right\}
$$

The total orderings ${ }^{0.6} \mathrm{O}$ that are compatible with the pairs in the crisp relation ${ }^{0.6} S$ is 1 out of the above 4 sets, that is:

$$
{ }^{0.6} O=\left\{\left\{a_{3}, a_{2}, a_{5}, a_{4}, a_{1}\right\}\right\}
$$

finally, ${ }^{1} \mathrm{O} \cap{ }^{0.9} \mathrm{O} \cap{ }^{0.8} \mathrm{O}^{0.6} \mathrm{O}={ }^{0.6} \mathrm{O}=\left\{\left\{a_{3}, a_{2}, a_{5}, a_{4}, a_{1}\right\}\right\}$

\section{Results}

A single crisp total ordering is achieved, in each of the two cases, as the group optimal preference ordering. In the first case $\left\{a_{3}, a_{5}, a_{2}, a_{4}, a_{1}\right\}$ with $0.6(60 \%)$ level of group agreement while in the second case is $\left\{a_{3}, a_{2}, a_{5}, a_{4}, a_{1}\right\}$ with 0.6 $(60 \%)$ level of group agreement as well.

It can be remembered that $P=a_{1}, C S=a_{2}, B=a_{3}, M=a_{4}$ and $F=a_{5}$, then, the ordering means;

The group optimal preference ordering (case 1) $=\{$ Business, Farming, Civil Service, Marriage, Politics $\}$ and

The group optimal preference ordering (case 2) $=\{$ Business, Civil Service, Farming, Marriage, Politics $\}$

\section{Discussion and Analysis}

It can be seen that the final result, in each case, reflected the collective opinion of the graduates with $60 \%$ level of agreement.

Business happened to be the first option for the group while Politics and Marriage comes as the last and second to the last alternatives, respectively, leaving Civil Service and Farming interchanging as the second and third alternatives. It can be analysed that the preference ordering obtained, from the same individuals, in most cases are not exactly the same. The preference ordering $\mathrm{P}_{2}, \mathrm{P}_{4}, \mathrm{P}_{5}, \mathrm{P}_{6}, \mathrm{P}_{8}, \mathrm{P}_{9}, \mathrm{P}_{10}$ from the first case and $\mathrm{P}_{2}^{*}, \mathrm{P}_{4}^{*}, \mathrm{P}_{5}^{*}, \mathrm{P}_{6}^{*}, \mathrm{P}_{8}^{*}, \mathrm{P}_{9}^{*}, \mathrm{P}_{10}^{*}$ from the second case, respectively, are not the same with ordering $\mathrm{P}_{5}, \mathrm{P}_{9}, \mathrm{P}_{10}$ and $\mathrm{P}_{5}^{*}, \mathrm{P}_{9}^{*}, \mathrm{P}_{10}^{*}$ almost entirely different. The preference ordering $\mathrm{P}_{1}, \mathrm{P}_{3}, \mathrm{P}_{7}$ and $\mathrm{P}_{1}^{*}, \mathrm{P}_{3}^{*}, \mathrm{P}_{7}^{*}$ are found to be the same in this work.

As each ordering represented the opinion of a particular person, the expectation is, the orderings per any individual need to be the same, but, due to some factors they usually differed when the Shiruma approach is applied. This is because in Shiruma approach each decision maker has the chance to give a preference strength between any two alternatives based on the descriptions used in obtaining the data. This description is what make this approach more suitable than the other, therefore for one to achieved best optimal solution in such decision-making careful measures must be considered. Based on the results obtained in this work, it can be concluded that the following conditions are required in order to obtained reflective individual preference ordering in Shiruma approach, that is the decision maker(s);

- $\quad$ needs to fully understand the description for obtaining the data

- needs to give best descriptions in preferring between any two alternatives in comparison

- $\quad$ to make sure that the inputs are based on the partial or total ordered relations

The three orderings stated above, that almost completely differed, are due to the negligence with regard to some of the above conditions, and such deviations are not helping in data analysis.

In addition to the analysis above, the results obtained show how the thinking of our graduates changed due to experiences. In the recent years, graduates, usually, consider civil service as first option, and now because of economic and political reasons the youth are taking a different direction. Marriage, which comes as the second to the last options, is the basic of maintaining peaceful existence and creating good family life, but the youths showed that dealing first with economic problem determined the level of readiness for one to established family life as it is fund demanding. Politics is one of the most appealing carriers, now adays, but it is highly critical and full of violence and injustice. The decision makers involved in this work showed that they kept Politics as the last option possibly due to the mentioned reasons. The $60 \%$ degree of agreement showed how each of the individuals involved is satisfied with the results obtained.

\section{Conclusion}

Making decision is one of the fundamental parts of living as it is required in executing any plan. The influential factors affecting our decisions are almost all fuzzy in nature as they are vague/imprecise, as such, for us to have a better decision we must employ a better technique to handle these influential factors. 
Application of Shiruma approach in making group decision, as shown in this work, is the most appropriate technique in dealing with the vagueness and imprecision affecting our decisions. The research can be furthered by letting the decision be by stages which, collectively, gives one decision for all. This type of research work will help in determining the best technique using which the feeling and forecast of our youth can be seen and it will help the governing bodies to easily understand the life and feelings of their people.

\section{References}

i. $\quad$ Blin, J. M. [1974], Fuzzy relations in group decision theory. Journal of Cybernetics, 4(2), pp. 12-22.

ii. Blin, J. M. and A. B. Whinston [1973], Fuzzy sets and social choice. Journal of Cybernetics, 3(4), pp. 28-36.

iii. $\quad$ Dewey, John (1978), How We Think, pp. 177-356 in Middle Works, vol 6.

iv. G. J. Klir, Yuan B (1995) Fuzzy Sets and Fuzzy Logic: The Theory and Applications', Prentice Hall, Englewood Cliff, NJ.

v. Garg H (2016) A novel correlation coefficients between Pythagorean fuzzy sets and its applications to decision making processes.

vi. International Journal Intelligent Systems. 12(31):1234-1252

vii. Garg H (2018) Hesitant Pythagorean fuzzy sets and their aggregation operators in multiple-attribute decisionmaking.

viii. International Journal of Uncertain Quantifications 8(3):267-289

ix. Garg H (2018) Hesitant Pythagorean fuzzy Maclaurin symmetric mean operators and its applications to multiattribute decision making process. International Journal Intelligent Systems https://doi.org/10.1002/int.22067

$x . \quad$ [8] Garg H (2017) Confidence levels-based Pythagorean fuzzy aggregation operators and its application to decisionmaking process.

xi. Journal of Computational Mathematics 23(4):546-571

xii. Garg H (2018) Linguistic Pythagorean fuzzy sets and its applications in multi attribute decision making process. International Journal Intelligent Systems 33(6):1234-1263

xiii. Garg H (2018) Some methods for strategic decision-making problems with immediate probabilities in Pythagorean fuzzy environment. International Journal Intelligent Systems 33(4):687-712

xiv. Garg H (2018) A new exponential operational laws and their aggregation operators of interval-valued Pythagorean fuzzy information. International Journal Intelligent Systems 33(3):653-683

xv. K. T. Atanassov, Intuitionistic fuzzy sets, Fuzzy Sets and Systems 20 (1986) 87-96.

xvi. $\quad$ L. A. Zadeh, Fuzzy sets Information and Control 8 (1965) 338-353.

xvii. L. A. Zadeh, [1975], Fuzzy logic and approximate reasoning. Synthese, 30(1), pp. 407-428

xviii. Shimura, eds. [1975], Fuzzy Sets and Their Applications to Cognitive and Decision Processes. Academic Press, New York, pp. X-

xix. $\quad 39,77-96$.

xx. Wei G, Garg H, Gao H, Wei C (2018) Interval-valued Pythagorean fuzzy Maclaurin symmetric mean operators in multiple attribute decision making. IEEE Access 6(1):67866-67884

xxi. Yager RR, Abbasov AM (2013) Pythagorean membership grades, complex numbers and decision making. International Journal Intelligent Systems 28:436-452.

xxii. Zeng W, Li D, Yin Q (2019) Weighted interval-valued hesitant fuzzy sets and its application in group decision making.

xxiii. Int J Fuzzy Syst. https://doi.org/10.1007/s40815-018-00599-2

xxiv. Zeng W, Li D, Gu Y (2018) Note on the aggregation operators and ranking of hesitant interval-valued fuzzy elements.

xxv. Journal of Soft Computations. https://doi.org/10.1007/s00500-018-3445-x

xxvi. Zimmermann, H. J. [1987], Fuzzy Sets, Decision Making, and Expert Systems. Kluwer, Boston.

xxvii. Zimmermann, H. J., L. A. Zadeh and B. R. Gaines, eds. [1984], Fuzzy Sets and Decision Analysis. North-Holland, New York.

xxviii. Zimmermann, H. J. and P. Zysno [1980], Latent connectives in human decision making. Fuzzy Sets and Systems. 4(1), pp. 37-51. 